\title{
Review of Pharmacotherapy in the Treatment of the Catatonic Syndrome: \\ First-Line, Adjunctive/Combination and Novel Options
}

\section{Authors:}

David R. Spiegel M.D. I*,

Brendan T. Carroll M.D. ${ }^{2}$, Jenny Lee M.D. I,

Stephanie Le M.D. ${ }^{\text {I }}$

Nikhil Borra M.D. ${ }^{\text {I }}$

\section{Authors Note:}

I Department of Psychiatry and Behavioral Sciences, Eastern Virginia Medical School, Norfolk, VA, USA

2 Department of Psychiatry, Chillicothe VA Medical Center, Chillicothe, Ohio, USA, Ohio University Heritage College of Osteopathic Medicine, Athens, Ohio, USA

Dr. Spiegel is a member of the Speaker's Bureau for Allergen Pharmaceuticals.

The authors received no support that facilitated conduct of the work described in the article or the writing of the article itself.

*Corresponding author:

David R. Spiegel, M.D.

Department of Psychiatry and Behavioral

Sciences

Eastern Virginia Medical School

825 Fairfax Avenue

Norfolk, VA 23507, USA

Email: spiegedr@evms.edu

\section{Abstract:}

Catatonia is a clinical syndrome characterized by a range of psychomotor abnormalities that occur in the context of a wide variety of both psychiatric and medical conditions. It occurs in affective, psychotic, autistic, developmental and medical disorders. Catatonia may present with unusual stereotypies and medical comorbidities. Despite these various etiologies, treatment has been convergent on the gamma aminobutyric acid agonists (i.e., benzodiazepines), and glutamate antagonists, corresponding with the known pathophysiology on this syndrome. Treatment refractory and emergent cases are often referred to electroconvulsive therapy. The focus of this manuscript will be to review these known treatments in montherapy, adjunctive, combined, and potential novel pharmacotherapy.

\section{Key Words:}

Catatonia; Gamma aminobutyric acid;

Glutamate 


\section{Review of Pharmacotherapy in the Treatment of the Catatonic Syndrome: First-Line, Adjunctive/Combination and Novel Options}

Introduction:

Catatonia has been associated with a number of medical conditions, including central nervous structural damage, and it has a reported incidence in roughly $10-15 \%$ of acutely ill psychiatric patients ${ }^{1}$. Although many theories of the pathophysiology of catatonia have been described, the exact mechanism is not clear. The catatonic presentation appears to be produced in states of glutamate and gamma aminobutyric acid (GABA) imbalance. It has been suggested that a decrease in GABAergic tone in the prefrontal areas result in a release of supplementary motor areas and lead to glutamate disinhibition. Catatonic symptoms present in episodes of glutamate hyperactivity and accordingly may resolve with decreased glutamate activity. ${ }^{1}$ Positive responses in catatonic patients to specific pharmacological therapy, including benzodiazepines and amantadine, favor the GABA-glutamate balance rationalization ${ }^{2,3}$.

\section{First-Line Treatments:}

Current pharmacological treatment strategies and theories behind the pathophysiology of catatonia have co-developed. Initially when catatonia was described, it was noted that symptoms were sensitive to benzodiazepines and other GABA agonists such as zolpidem, which demonstrated the role of GABA in the syndrome ${ }^{4}$. The proposed medical treatment algorithm for catatonia reflects the cumulative experience in this area and features the lorazepam test (by mouth, intravenous, or intramuscular administration of lorazepam 1-2 $\mathrm{mg}$ ) for the rapid, albeit transient, resolution of acute catatonia. If improvement is seen after the challenge test, treatment with increasing doses of lorazepam/benzodiazepines is recommended ${ }^{5}$. First-line treatment of catatonia continues to be benzodiazepines, specifically lorazepam, and electroconvulsive therapy (ECT) for refractory cases, of catatonias in the frontosubcortical motor loop ${ }^{6}$. The response to benzodiazepines is dose-dependent. Typically, lorazepam is started at 8-24 mg per day, then slowly titrated up, while remaining vigilant of potential sedative side effects. Changes are often noted in less than 7 days $^{7}$, with a response rate of $60-80 \%{ }^{8}$. Systematic studies of catatonia confer that approximately $80 \%$ of recognized cases respond to benzodiazepine treatment, leaving fewer than $20 \%$ requiring referral for ECT or other treatment options?.

ECT can be an effective treatment for catatonia. This was recently discussed by optimizing ECT for catatonia. Unilateral electrode placement, brief currents, low total stimulus charge and twice weekly treatments are often offered. But when these parameters are followed then ECT for catatonia, they are likely to fail to relieve the catatonic syndrome. Fink et al recommended treatments administered daily in febrile, dehydrated, and overly excited delirium. These patients bitemporal electrode placement with basic threshold testing may offer the best outcome. Fear of memory loss can be balanced against reversing the negativism, and delirium, to ensure patient survival. No physician can anticipate how many treatments are best estimate. Continuation ECT is necessary for all patients until the patient returns to preillness state $^{9}$.

Atypical Antipsychotics, Non-Benzodiazepine First Generation Anticonvulsants:

Dopaminergic involvement in catatonia has been brought to light by the successful use of antipsychotics in the treatment of catatonia ${ }^{10}$. However, the inconclusive literature on antipsychotics has led to an expert consensus favoring initial treatment with lorazepam and consideration of ECT for refractory or severely compromised cases if lorazepam fails after a period of days. If antipsychotics are administered to treat catatonia, a cautious trial of these agents with continued benzodiazepines and careful monitoring for worsening catatonia or signs of NM is recommended ${ }^{8}$.

Additionally, the use of anticonvulsants, esp., valproic acid has been demonstrated to be 


\section{Review of Pharmacotherapy in the Treatment of the Catatonic Syndrome: First-Line, Adjunctive/Combination and Novel Options}

effective in treating catatonia. The mechanism is not clear on how valproic acid works in treating catatonia, but it is clear that it increases central nervous system GABA. Studies have shown that valproic acid enhances GABA level and neuronal GABA responsiveness ${ }^{11}$. Finally, carbamazepine has been reported to be an effective treatment, both in the acute phase and maintenance, of a subgroup of patients with retarded catatonia. While the authors did not speculate the pharmacodynamics of the medication response ${ }^{12}$, carbamazepine does seem to stimulate GABA-ergic transmission as well as its proposed primary action of binding to a receptor near or at sodium channels ${ }^{13}$.

Zolpidem: More Than Just a NonBenzodiazepine Hypnotic

Zolpidem is also a GABA-A receptor positive allosteric modulator, similar to benzodiazepines, although it displays a high affinity to alpha 1GABA (A) receptors, an intermediate affinity to alpha(2)- and alpha(3)-GABA(A) receptors and fails to bind to alpha(5)-GABA(A) receptors ${ }^{14}$. Additionally, there have been reports that zolpidem and its intermediates has been shown to protect hippocampal neurons from glutamateinduced death in rat hippocampal cells treated with glutamate $10 \mathrm{mM}^{15}$. Similar to the lorazepam challenge to help clinically diagnose catatonia, zolpidem challenge test can be used as a second-line agent if there is inadequate response to the lorazepam challenge test. Following zolpidem (5mg-10mg) administration with peak effect at 1-2 hours, there are reports of significant transient improvement of catatonic features, only to return to premorbid catatonia by $5-6$ hours post administration ${ }^{16}$.

In a case report of a woman in a catatonic state secondary to a subcortical stroke, dramatic improvement with zolpidem was noted ${ }^{17}$. In an open study, the same group quantified dosage and timing of response to zolpidem. Of the seven patients, catatonic symptoms were arrested within 30 minutes of ingestion at a plasma concentration that ranged from 80 to 130 $\mathrm{ng} / \mathrm{L}^{18}$. Reports estimated a lasting effect between 2-5 hours. These findings were congruent with a later study conducted by the same group, where between a plasma level of $80-150 \mathrm{ng} / \mathrm{L}$, there was a $50 \%$ reduction of symptoms noted within 20 minutes of ingestion of $10 \mathrm{mg}$ of zolpidem ${ }^{19}$. Zolpidem has a shortlife and low side-effect profile, however it is a time- and concentration-dependent inhibitor of CYP3A ${ }^{20}$.

Interestingly, some individuals who experience severe brain damage are left with disorders of consciousness. While they can appear to be awake, these individuals lack awareness of their surroundings and cannot respond to events going on around them. The observation that zolpidem can produce paradoxical recovery of speech, cognitive and motor functions in select subjects with severe brain injury, offers a potential interesting corollary to patients with retarded catatonia that respond to zolpidem ${ }^{21}$. These phenomenologic and treatment response similarities should be broached with caution.

The commonly recognized diagnostic features of (retarded) catatonia include immobility and mutism, but patients with catatonia notably display many other signs and may not be mute or immobile. Stuporous patients (as those described above), for example, are often mute, although stuporous and mute patients are not all catatonic; however, definitions of stupor in catatonia that are based on the simultaneous presence of other catatonic features skirt the issue of directly defining stupor. Attempts to discriminate between stupor related to a psychiatric condition and stupor caused by a recognized neurological disease have not been helpful, given recent findings of brain changes in catatonic patients who respond well to lorazepam or electroconvulsive therapy. It has been proposed that stupor may be defined as a state of unresponsiveness from which the patient can be aroused only by vigorous and repeated stimuli and back into which the patient lapses as soon as the stimuli cease. This description is consistent with the literature on catatonia. Ultimately, (retarded) catatonic patients are generally aware of their environment, although not able to necessarily express this during an 


\section{Review of Pharmacotherapy in the Treatment of the Catatonic Syndrome: First-Line, Adjunctive/Combination and Novel Options}

acute state $^{22}$. Thus, to intimate that these 2 clinical entities should be considered as "catatonia," would dramatically broaden the prevalence of catatonic syndrome and potentially confuse the stupor of delirium from the immobile and mute state of catatonia ${ }^{23}$.

Regardless, a possible explanation of the above considers that extensive damage to the cortex can lead to the loss of pathways between cortical regions, and between cortical and subcortical areas. One such pathway consists of excitatory projections from the cortex to a subcortical structure called the striatum, which in turn sends inhibitory projections to a region called the globus pallidus. When not inhibited by the striatum, the globus pallidus inhibits the thalamus. The net effect is that loss of excitatory projections from the cortex after a severe brain injury can indirectly result in inhibition of the thalamus. Removing this inhibition is critical for restoring normal brain function because the thalamus is a major source of arousal inputs to the cortex. Zolpidem, known to be selective for a particular subtype of GABA receptors (GABA(A) alpha 1) which are expressed on inhibitory neurons in the globus pallidus. Therefore, it has been proposed that zolpidem blocks the inhibitory inputs from this structure to the thalamus, thus allowing the thalamus to excite the cortex and help restore cognitive and motor functions ${ }^{24}$.

N-methyl-D-aspartate Receptor Antagonists: Amantadine, Memantine

In some benzodiazepine-resistant cases and although randomized trials of alternatives have not been performed, N-methyl-D-aspartate (NMDA) receptor antagonists, including amantadine (initial dose $100 \mathrm{mg}$ three times a day, up to $500 \mathrm{mg}$ three times a day reported) and memantine (initial dose $5 \mathrm{mg} /$ day, up to 20 $\mathrm{mg} /$ day reported) may provide benefit. The evidence of amantadine is somewhat more established than its derivative, memantine. Amantadine's effect has been speculated to occur on hypoactive receptors on inhibitory GABAergic interneurons in the prefrontal cortex and hippocampus ${ }^{25}$.

Carroll and colleagues found an improvement within seven days of administration of either amantadine or memantine ${ }^{3}$.. These findings were confirmed in over ten subsequent case reports. The same group proposed that amantadine enhances dopaminergic activity by working at both pre- and post-synapses ${ }^{6}$. Gianutsos and group confirmed that amantadine increases the synthesis and release of dopamine as well as inhibiting the reuptake; it also appeared to play a role in the affinity of post-synaptic receptors ${ }^{26}$. In a case report of an ECT-resistant catatonia, amantadine was also found to be effective ${ }^{27}$. Amantadine reaches peak plasma concentrations in less than 4 hours, with a plateau by 72 hours in the absence of a loading dose ${ }^{28}$. Typically in the setting of a traumatic brain injury, patients are prescribed $400 \mathrm{mg}$ per day in adults ${ }^{29,30}$. Vargus-Adams and colleagues found similar pharmacokinetics of amantadine when studying its effect on six children and adolescents with brain injury. Amantadine was tolerated with a low side-effect profile at $6 \mathrm{mg} / \mathrm{kg} / \mathrm{day}^{31}$. In a case report of a 92-year-old woman with the development of catatonia due to (recurrent) major depressive disorder without psychotic features, adjunctive memantine ( $5 \mathrm{mg} /$ day) to low dose lorazepam $(0.5 \mathrm{mg}, 2 \mathrm{x} /$ day $)$ resulted in resolution of catatonic symptoms, 24 hours after the former was added ${ }^{32}$.

Indirect N-methyl-D-aspartate Receptor Antagonists: Topiramate, Levetericetam

While much less studied than valproic acid and carbamazepine and their reported successes in the treatment of catatonia, topiramate has also been reported in a case series to successfully treat catatonia. Topiramate, a "broad spectrum" anticonvulsant, has multiple mechanism of action, although most relevant for this discussion, it is postulated as an AMPA (+/kainite) receptor antagonist may attenuate NMDA receptor function ${ }^{33}$. Lastly, there is a report in the literature of the successful treatment of excited catatonia in a patient with acute mania due to Bipolar Disorder with levetiracetam ${ }^{34}$. Whether thru its binding 


\section{Review of Pharmacotherapy in the Treatment of the Catatonic Syndrome: First-Line, Adjunctive/Combination and Novel Options}

specifically to the vesicle protein, SV2A, located in a presynaptic site (and involved in neurotransmitter release) or thru its ability to block P/Q-type voltage dependent calcium channels, levetiracetam has been shown to decrease glutamate release offering an explanation as to the utility of levetiracetam in this case ${ }^{35}$. Alternatively, there has also been a case of catatonia precipitated by levetiracetam administration $^{36}$.

Novel Treatment Options: $N$-acetylcysteine (NAC)

Lastly, the authors are familiar with using $N$ acetylcysteine (NAC) as a potential emerging as agent in the treatment of catatonia. Like many therapies, the clinical origins of NAC are far removed from its current use in psychiatry. Whereas the mechanisms of NAC are only beginning to be understood, it is likely that NAC is exerting benefits beyond being a precursor to the antioxidant, glutathione, modulating glutamatergic, neurotropic and inflammatory pathways.

It has been proposed that cysteine assists in the regulation of neuronal intra- and extracellular exchange of glutamate through the cystineglutamate antiporter. Whereas this antiporter is ubiquitous throughout all cell types, in the brain it is preferentially located on glial cells. The dimer, cystine, is taken up by astrocytes and exchanged for glutamate, which is released into the extracellular space. This free glutamate appears to stimulate inhibitory metabotropic glutamate receptors on glutamatergic nerve terminals and thereby reduce the synaptic release of glutamate. Given the accentuated activity of glutamate on the NMDA receptor in catatonia, NAC appears to be a promising therapeutic target and provides a window of treatment opportunity in a field where current treatments are limited ${ }^{37}$.

Alternatively, the cellular localization of cystine-glutamate antiporters on glial cells neighboring neurons and the extrasynaptic location of mGluR $2 / 3$ on axon terminals pose the possibility that these systems influence one another. In support, earlier studies have shown that blockade of mGluR2/3 produces an increase in extracellular levels of glutamate and dopamine, implying the existence of endogenous stimulation of these receptors that is capable of modulating synaptic activity. As above, cystineglutamate antiporters are a source of glutamate supplying endogenous stimulation to mGluR2/3. In support and consistent with recent reports showing that nonvesicular glutamate release in vitro regulates glutamatergic synaptic transmission, blockade of the antiporter has been demonstrated to prevent the rise in extracellular glutamate associated with mGluR2/3 blockade. Blockade of the antiporter also increased extracellular dopamine levels, which are well characterized to be of vesicular, synaptic origin. Moreover, the increase in dopamine has been shown to be reversed by stimulating mGluR $2 / 3$, indicating that glutamate derived from cystineglutamate exchange is providing tone to presynaptic mGluR2/3 heteroreceptors. Given that hypodopaminergia is posited in the pathogenesis of catatonia, NAC may be effective in the treatment of the latter thru regulation of dopaminergic tone ${ }^{38}$.

\section{Combination Treatment}

Support for GABA and glutamate dysfunction in the catatonic syndrome is highlighted by the successful use of anti-epileptics known to be GABAergic as well as indirectly antagonistic of AMPA glutamate receptors, as adjuvant therapy in cases of catatonia that did not respond to GABA agonists alone ${ }^{39,40}$. In addition, it is thought that catatonia results form an imbalance of GABA (GABA-A), Dopamine (D2) and glutamate (NMDA). We have previously published on the pharmacotherapy of catatonia. However, we have not discussed combined treatment. Recently, a case of excited catatonia was reported of benzodiazepine-reported treated with a combination of memantine and divalproex $^{41}$. Since the ICD-10 code (F06.1:Catatonic disorder due to known physiological condition) has only been recently adopted, one of the authors (B.T.C.) chose to anecdotally search for combination treatments on all patients treated at the Chillicothe VA 


\section{Review of Pharmacotherapy in the Treatment of the Catatonic Syndrome: First-Line, Adjunctive/Combination and Novel Options}

Medical Center, Chillicothe, Ohio, USA, from October 1, 2015 to July 31, 2016 with a primary or secondary ICD-10 code of F06.1. These patients were identified and their pharmacologic treatments were reviewed. While on mood stabilizers, antidepressants, first generation and second generation antipsychotics, this author looked at the medication classes that affected of GABA-A , Dopamine (D2) and glutamate (NMDA) transmission. Twelve cases, and in 6 of these a combination of these medications, were found and used concomitantly. There was a 7 th case with a combination of memantine and divalproex, in which lorazepam had been tapered. This suggests that in some settings clinicians may use a combination of medications to treat catatonia.

The exact neurobiology of catatonia is yet to be described and is suspected to differ on the basis of whether underlying etiology is due to a general medical condition (GMC), a preexisting psychiatric condition, or a drug induced catatonia. This suspicion is based on differences to response to pharmacologic agents as catatonia secondary to a psychiatric conditions tends to respond better following treatment with antipsychotics while this same treatment strategy tends to induce or worsen catatonia of a $\mathrm{GMC}^{24}$. What has become somewhat clear through the successful use and combination of agents targeting the GABAergic, glutamatergic, and dopaminergic systems, is that catatonia following a GMC such as a TBI likely involves diffuse dysregulation of multiple neurochemical pathways, rather than focal injury or dysfunction $^{42}$. The normal time course and response to treatment has been observed to differ widely on a case basis. This variation appears to again be linked to basis of the etiology of the catatonia. Catatonia linked to a GMC tends to be exquisitely responsive to GABA agonists often resolving and relapsing on the basis of therapeutic dosing and tends to follow this pattern until the patients GMC has resolved ${ }^{38}$. Cases with a psychiatric etiology however tend to be more chronic in nature and response follows adequate control of psychiatric symptoms $^{42}$.

Recognition of catatonia is imperative before treatment can be initiated. First line treatment should continue to be GABA agonists/benzodiazepines such as lorazepam although more severe or treatment refractory cases also respond excellently to ECT. Nonetheless, in those cases which do not fully respond to the above, the above discussed medications, including zolpidem and antiglutamatergic medications, may be utilized as adjunctive treatment or in certain cases, monotherapy. 


\section{Review of Pharmacotherapy in the Treatment of the Catatonic Syndrome: First-Line, Adjunctive/Combination and Novel Options}

\section{$\underline{\text { References }}$}

1. Spiegel DR, Klaiber N. A Case of Catatonia Status-Post Left Middle Cerebral Artery Cerebrovascular Accident, Treated Successfully With Olanzapine. Clin Neuropharmacol. 2013;36(4):135-137. doi:10.1097/WNF.0b013e3182956d4d.

2. Babington PW, Spiegel DR. Treatment of catatonia with olanzapine and amantadine. Psychosomatics. 2007;48(6):534-536. doi:10.1176/appi.psy.48.6.534.

3. Carroll BT, Goforth HW, Thomas C, et al. Review of adjunctive glutamate antagonist therapy in the treatment of catatonic syndromes. J Neuropsychiatry Clin Neurosci. 2007;19(4):406-412. doi:10.1176/appi.neuropsych.19.4.406.

4. Thomas P, Rascle C, Mastain B, Maron M, Vaiva G. Test for catatonia with zolpidem. Lancet (London, England). 1997;349(9053):702. doi:10.1016/S01406736(05)60139-0.

5. Dhossche D, Wilson C, Wachtel L. Catatonia in Childhood and Adolescence: Implications for the DSM-5. Prim Psychiatry. 2010:1723-1726.

6. Northoff G. Catatonia and neuroleptic malignant syndrome: psychopathology and pathophysiology. I Neural Transm. 2002;109:1453-1467. doi:10.1007/s00702-002-0762-z.

7. Fink M, Taylor M. Neuroleptic malignant syndrome is malignant catatonia, warranting treatments efficacious for catatonia. Prog Neuropsychopharmacol Biol. 2006 Aug 30;30(6):1182-3; author reply 1184-5.
8. Francis A. Catatonia: Diagnosis, Classification, and Treatment. Curr Psychiatry Rep. 2010;12(3):180-185. doi:10.1007/s11920-010-0113-y.

9. Fink M, Kellner $\mathrm{CH}$, McCall WV. Optimizing ECT Technique in Treating Catatonia. J ECT. 2016;32(3):149-150. doi:10.1097/YCT.0000000000000345.

10. Chang CH, Hsiao YL, Hsu CY, Chen ST. Treatment of catatonia with olanzapine: a case report. Prog Neuropsychopharmacol Biol Psychiatry. 2009 Nov 13;33(8):1559-60.

11. Bowers R, Ajit SS. Is there a role for valproic acid in the treatment of catatonia? $J$ Neuropsychiatry Clin Neurosci. $\quad 2007 ; 19(2): 197-198$. doi:10.1176/jnp.2007.19.2.197.

12. Kritzinger PR, Jordaan GP. Catatonia: an open prospective series with carbamazepine. Int $J$ Neuropsychopharmacol. 2001;4(3):251257. doi:doi:10.1017/S1461145701002486.

13. Hosák L, Libiger J. Antiepileptic drugs in schizophrenia: a review. Eur Psychiatry. 2002;17(7):371-378.

14. Crestani F, Martin JR, Möhler H, Rudolph U. Mechanism of action of the hypnotic zolpidem in vivo. $B r \quad J$ Pharmacol. 2000;131(7):1251-1254. doi:10.1038/sj.bjp.0703717.

15. García-Santos G, Herrera F, Martín V, et al. Antioxidant activity and neuroprotective effects of zolpidem and several synthesis intermediates. Free Radic Res. 2004;38(12):1289-1299. doi:10.1080/10715760400017343. 


\section{Review of Pharmacotherapy in the Treatment of the Catatonic Syndrome: First-Line, Adjunctive/Combination and Novel Options}

16. Bastiampillai T, McGovern V, Lloyd B, Hittur Lingappa S, Nelson A. Treatment refractory chronic catatonia responsive to zolpidem challenge. Aust $N \quad Z \quad J$ Psychiatry. 2016;50(1):98. doi:10.1177/0004867415582232.

17. Mastain B, Vaiva G, Guerouaou D, Pommery J. Favourable effect of zolpidem on catatonia. Rev Neurol (Paris). 1995 Jan;151(1):52-6.

18. Rasele C, Thomas P, Maron M, et al. Catatonia relief with zolpidem: An open study. Eur Neuropsychopharmacol. 1997;7:S277. doi:10.1016/S0924977X(97)88920-1.

19. Thomas P, Cottencin O, Rascle C, Vaiva G. A case of agitated catatonia. Pharmacopsychiatry. 1999 Jan;32(1):3840.

20. Polasek T, Sadagopal J, Elliot D. In vitro-in vivo extrapolation of zolpidem as a perpetrator of metabolic interactions involving CYP3A. Eur $J$ Clin Pharmacol. 2010 Mar;66(3):275-83.

21. Williams ST, Conte MM, Goldfine AM, et al. Common resting brain dynamics indicate a possible mechanism underlying zolpidem response in severe brain injury. Elife. 2013;2:e01157. doi:10.7554/eLife.01157.

22. Sallin K, Lagercrantz H, Evers K, Engström I, Hjern A, Petrovic P. Resignation Syndrome: Catatonia? Culture-Bound? Front Behav Neurosci. 2016;10:7. doi:10.3389/fnbeh.2016.00007.

23. Taylor MA, Fink M. Catatonia in psychiatric classification: a home of its own. Am J Psychiatry. 2003;160(7):1233-1241. doi:10.1176/appi.ajp.160.7.1233.

24. Akeju O, Brown EN, Brefel-Courbon C, et al. Awakened by a sleeping pill. Elife. 2013;2:e01658. doi:10.7554/eLife.01658.

25. Coyle J. The GABA-glutamate connection in schizophrenia: which is the proximate cause? Biochem Pharmacol. 2004 Oct 15;68(8):1507-14.

26. Gianutsos G, Chute S, Dunn J. Pharmacological changes in dopaminergic systems induced by longterm administration of amantadine. Eur $J$ Pharmacol. 1985 Apr 16;110(3):357-61.

27. Goetz M, Kitzlerova E, Hrdlicka M. Combined use of electroconvulsive therapy and amantadine in adolescent catatonia precipitated by cyber-bullying. J Child Adolesc Psychopharmacol. 2013 Apr;23(3):228-31.

28. Bleidner WE, Harmon JB, Hewes WE, Lynes TE, Hermann EC. Absorption, distribution and excretion of amantadine hydrochloride. J Pharmacol Exp Ther. 1965;150(3):484-490.

29. Gualtieri $\mathrm{T}$, Chandler $\mathrm{M}$, Coons $\mathrm{T}$. Amantadine: a new clinical profile for traumatic brain injury. Clin Neuropharmacol. 1989 Aug;12(4):25870.

30. Zafonte RD, Lexell J, Cullen N. Possible applications for dopaminergic agents following traumatic brain injury: part 2. $J$ Head Trauma Rehabil. 2001 Feb;16(1):112-6.

31. Vargus-Adams J, McMahon M, Michaud L, Bean J. Pharmacokinetics of amantadine in children with impaired consciousness due to acquired brain injury: preliminary findings using a sparse-sampling technique. $P M \& R$. 


\section{Review of Pharmacotherapy in the Treatment of the Catatonic Syndrome: First-Line, Adjunctive/Combination and Novel Options}

2010 Jan;2(1):37-42.

32. Obregon DF, Velasco RM, Wuerz TP, Catalano MC, Catalano G, Kahn D. Memantine and catatonia: a case report and literature review. J Psychiatr Pract. 2011;17(4):292-299.

doi:10.1097/01.pra.0000400268.60537.5 e.

33. McDaniel WW, Spiegel DR, Sahota AK. Topiramate effect in catatonia: a case series. J Neuropsychiatry Clin Neurosci. 2006;18:234-238.

doi:10.1176/appi.neuropsych.18.2.234.

34. Muneer A. Catatonia in a patient with bipolar disorder type I. J Neurosci Rural Pract. 2014;5(3):314-316. doi:10.4103/0976-3147.133652.

35. Lee C-Y, Chen C-C, Liou H-H. Levetiracetam inhibits glutamate transmission through presynaptic P/Qtype calcium channels on the granule cells of the dentate gyrus. $B r \quad J$ Pharmacol. 2009;158(7):1753-1762. doi:10.1111/j.1476-5381.2009.00463.x.

36. Sienaert P, Dhossche DM, Vancampfort D, De Hert M, Gazdag G. A clinical review of the treatment of catatonia. Front Psychiatry. 2014;5:181. doi:10.3389/fpsyt.2014.00181.

37. Dean O, Giorlando F, Berk M. Nacetylcysteine in psychiatry: current therapeutic evidence and potential mechanisms of action. $J$ Psychiatry Neurosci. 2011;36(2):78-86. doi:10.1503/jpn.100057.

38. Baker DA, Xi Z-X, Shen H, Swanson CJ, Kalivas PW. The origin and neuronal function of in vivo nonsynaptic glutamate. $J$ Neurosci. 2002;22(20):9134-9141.

39. Munoz C, Yulan N, Achaval V, Appiani $\mathrm{F}$, Carroll BT. Memantine in major depression with catatonic features. $J$ Neuropsychiatry Clin Neurosci. 2008;20(1):119-120. doi:10.1176/jnp.2008.20.1.119.

40. Yoshida I, Monji A, Hashioka S, et al. Prophylactic effect of valproate in the treatment for siblings with catatonia: a case report. J Clin Psychopharmacol. 2005 Oct;25(5):504-5.

41. Soltanianzadeh Y, Greene E, Slootsky V. A 32-Year-Old Man with Chest Pain, Confused Mental State, and Muscle Rigidity. Psychiatr Ann. 2016;46(5):274276. doi:10.3928/00485713-2016031501.

42. Spiegel DR, Thanam S., and Rayamajhi U. In: Catatonia on the Consultation Liaison Service and Other Clinical Settings. Ed. Carroll BT and Spiegel DR., Nova Science Publishers, New York, 2016. 